\title{
Therapeutic Targeting of Hepatic Macrophages
}

\author{
Hilde Nijland ${ }^{1} \cdot$ Ruchi Bansal ${ }^{1}$ (I)
}

Published online: 24 April 2020

(C) The Author(s) 2020

\begin{abstract}
Purpose of Review This review outlines the current knowledge about hepatic macrophages and provides an overview of therapeutic approaches to target hepatic macrophages for the treatment of liver diseases.

Recent Findings In recent years, it has been increasingly recognized that hepatic macrophages (resident macrophages, Kupffer cells, or circulating bone marrow monocyte-derived macrophages) are implicated in liver homeostasis as well as in disease progression and resolution. More recently, different populations of hepatic macrophages with distinct phenotypes and functions have been identified that have shown to play distinct roles in the pathogenesis of various acute and chronic liver diseases. The understanding of the role of hepatic macrophages in initiation, progression, and resolution of liver diseases has given rise to the development of therapeutics that can target different phenotypes of hepatic macrophages. Innovative strategies comprises of microRNA (miRNA), small interfering RNA (siRNA), therapeutic proteins, and small-molecule inhibitors.

Summary Evidence from recent in vitro and in vivo studies support the fact that hepatic macrophages can be efficiently targeted using miRNA/siRNA-based approaches, protein-based approaches, and small-molecule inhibitors for the treatment of liver diseases. However, more in-depth understanding underlying the roles of distinct macrophage phenotypes in different liver diseases is required for the translation of novel targeted therapeutics to the clinic.
\end{abstract}

Keywords Hepatic macrophages $\cdot$ Targeted therapeutics $\cdot$ Liver diseases $\cdot$ Monocytes $\cdot$ Kupffer cells

\section{Introduction}

The liver, the largest organ in the human body, exerts multiple vital bodily functions including blood detoxification, bile production, and synthesis, storage, and redistribution of different biomolecules like lipids, proteins, and carbohydrates $[1,2]$. The liver also plays an important role in maintaining liver immune homeostasis and employs different mechanisms to suppress immune system and create immune tolerance [1-3]. Numerous studies have shown that hepatic macrophages [resident Kupffer cells (KCs) and monocyte-derived macrophages (MoMFs)] represent a diversified population of immune cells that are derived from varied sources and play a

This article is part of the Topical Collection on Chronic Liver Disease

Ruchi Bansal

r.bansal@utwente.nl

1 Translational Liver Research, Department of Medical cell Biophysics, Technical Medical Centre, Faculty of Science and Technology, University of Twente, Drienerlolaan 5, 7522 NB Enschede, The Netherlands pivotal (and distinct) role in the initiation, progression, and restoration of liver diseases [4]. In recent years, incredible heterogeneity in these hepatic macrophages has been revealed, highlighting their complexity and involvement in liver diseases [2, 5-8]. While KCs maintain liver homeostasis, acute liver damage can result in activation of KCs into inflammatory phenotypes and instigate rapid infiltration of circulating monocytes into the injured liver resulting in intrahepatic macrophage imbalance. This imbalance results in increased liver inflammation, which can eventually deteriorate normal liver functions $[8,9]$. Hepatic macrophages also engage closely with parenchymal (hepatocytes) and non-parenchymal cells, namely, hepatic stellate cells (HSCs) and liver sinusoidal endothelial cells (LSECs). HSCs are perisinusoidal cells located in a space between the LSECs and hepatocytes, called the space of Disse, and exert multiple functions such as vitamin A storage, immune-regulation, and remodeling of extracellular matrix [10-12]. Liver injury causes hepatic macrophages to release several inflammatory cytokines and chemokines that can activate these HSCs, thereby fostering not only inflammation but also liver fibrogenesis characterized by an excessive accumulation of ECM proteins, mainly produced 
by activated HSCs, leading to formation of scar tissue with distorted liver architecture and loss of liver function [13, 14]. This review presents the brief overview of hepatic macrophages and discusses the advancements that have been made in their understanding that has led to novel therapies that are currently been examined in preclinical models and clinical trials. Promising therapeutic approaches that have been summarized in this review provide new perspectives towards the effective and efficient treatment of liver diseases.

\section{Origin and Heterogeneity of Hepatic Macrophages}

The liver comprises of about $80-90 \%$ of the macrophages in the body [7]. Hepatic macrophages originate from different sources, resulting in cellular heterogeneity in the liver [2]. Hepatic macrophages can be derived from resident hepatic macrophages (KCs) or from distinct populations of infiltrating macrophages, i.e., bone marrow (BM)-derived macrophages, avascular peritoneal macrophages (PMs) that reside in subcapsular regions of the liver, and/or splenic monocytes [2, 4, 9, $15,16]$. Resident hepatic macrophages, the so-called KCs, are non-migrating and self-renewing cells located at the luminal side of the hepatic sinusoidal endothelium $[15,17]$. Owing to hepatic metabolic or toxic insults, circulating monocytes/ macrophages known as bone marrow monocyte-derived macrophages (MoMFs) rapidly infiltrate the liver tissue [6].

Traditionally, macrophages depending on microenvironmental signals have been classified into two distinct subgroups referred to as classically-activated M1 and alternatively activated M2 macrophages $[18,19]$. Macrophages can be polarized towards the pro-inflammatory (and antitumorigenic) M1 phenotype in response to lipopolysaccharides (LPS) and interferon gamma (IFN- $\gamma$ ) stimulation, secreting high levels of pro-inflammatory cytokines. Alternatively, macrophages can be polarized towards restorative or antiinflammatory (and pro-tumorigenic) M2 phenotype in response to IL-4 and IL-13 stimulation. In contrast to M1, M2 macrophages suppress inflammatory responses and facilitate tissue repair $[18,20]$. M2 macrophages can be further subclassified into M2a, M2b, M2c, and M2d based on different stimuli, distinct gene expression profiles, and functions $[21,22]$. However, recent studies have identified tremendous diversity in macrophage phenotypes, attributed to their plasticity, capable of adapting their phenotype in response to diverse microenvironmental as reviewed extensively elsewhere $[4,5]$. It is therefore imperative to gain mechanistic insights into these distinct phenotypes of macrophages to develop smart approaches to target selectively pathogenic phenotype of macrophages for the treatment of pathological diseases without affecting other phenotypic macrophages.

\section{Role of Hepatic Macrophages in Liver Diseases}

Hepatic macrophages are important determinant in the pathogenesis of liver diseases. They play a central role in promoting inflammation, the hallmark of nearly all liver diseases including acute liver failure (ALF), liver fibrosis, non-alcoholic fatty liver disease (NAFLD)/non-alcoholic steatohepatitis (NASH), alcoholic liver disease (ALD), viral hepatitis, and hepatocellular carcinoma (HCC) [2]. When liver injury ensues, triggered by multiple factors including alcohol/drug abuse, viral infections, fatty diet, or auto-immune disorders [23], hepatocytes are injured and release mediators leading to the activation of resident KCs. This results in the production of inflammatory cytokines and chemokines, e.g., $\mathrm{C}-\mathrm{C}$ motif chemokine ligands (CCLs) that induce massive infiltration of inflammatory monocytes into the injured liver, leading to higher levels of secreted cytokines and chemokines promoting chronic inflammation [9]. The role of hepatic macrophages in different liver diseases has been extensively reviewed elsewhere $[2,8$, 9, 15].

As mentioned earlier, hepatic macrophages interact with HSCs, whereby, upon activation, HSCs undergo functional and morphological changes resulting in proliferative myofibroblasts that produce excessive ECM and secrete numerous pro-inflammatory and pro-fibrotic factors [12, 24]. Since it is known that these events promote inflammation and contribute to liver fibrogenesis, HSCs are considered to be the main effector cells in hepatic fibrosis [25]. It has also been shown that these myofibroblasts can differentiate hepatic macrophages into pro-inflammatory and pro-fibrotic phenotypic macrophages [26], therefore playing a central role in liver fibrogenesis together with hepatic macrophages.

The plasticity and the capability of hepatic macrophages to switch phenotypes according to microenvironmental signaling account for their numerous functions in liver diseases. Because of this, manifold challenges remain in understanding the full spectrum of mechanisms of hepatic macrophages and their activation, recruitment, and involvement in promoting liver disease progression or in restoring damaged liver tissue [9]. This knowledge will open promising opportunities towards the treatment of liver diseases.

\section{Therapeutic Targeting of Hepatic Macrophages}

Over past years, due to the growing knowledge about hepatic macrophages, multiple mechanisms and pathways have been identified and investigated that regulate their recruitment, activation, differentiation, and polarization, based on which, a number of novel therapies have been developed and examined in various (pre-)clinical studies [2]. These novel therapies to 
treat liver diseases mainly aim at targeting hepatic macrophages. Increasing evidence from mouse models and clinical studies in patients with liver diseases support the potential of hepatic macrophage targeting attributed to reduced inflammation thereby attenuation of liver fibrosis. Nowadays, targeting therapies are predominantly focused on (i) reducing the activation of KCs thereby reducing the release of proinflammatory mediators, (ii) promoting a switch from a pathogenic macrophage phenotype towards a restorative macrophage phenotype promoting disease resolution and liver regeneration, and/or (iii) reducing inflammatory monocyte recruitment into the injured liver to attenuate liver inflammation $[2,7,15]$.

However, treatment options for most liver diseases still remain limited, owing to several challenges that are hampering the development of potential therapeutics: (1) discrepancy in macrophage phenotypes in humans and in animal models resulting in poor translation of therapeutics from animal models to human patients; (2) greater heterogeneity in humans as compared with inbred mouse strains due to several intrinsic (genetics, ethnicity, sex, and age) and extrinsic (microbiota, infections, medications) factors; (3) inadequate knowledge about human macrophages as compared with mouse models. Importantly, macrophages display tremendous heterogeneity, as shown in animal models and human liver diseases, with distinct functions in disease initiation and progression as well as disease regression and homeostasis $[2,8,9,15]$. Therefore, it is crucial to target the pathogenic phenotypes of macrophages therapeutically without hindering the functions of socalled restorative or homeostatic macrophages [2].

We review multiple approaches that have been investigated for therapeutic targeting of hepatic macrophages. These approaches are divided into three categories as depicted in Table 1: (a) miRNA/siRNA-based approaches, (b) proteinbased approaches, and (c) small-molecule inhibitors. We have also discussed synthetic nanoparticles (NPs) and extracellular vesicles (EVs) as efficient nanocarriers of therapeutics. While NPs mainly comprises of liposomes, polymeric NPs, and organic or inorganic NPs, EVs are biologically formed membrane-derived vesicles that carry multiple bioactive molecules to regulate cellular responses [41, 42]. NPs and EVs can both be selectively engineered for the delivery of several therapeutics including miRNA/siRNA, proteins/peptides, and small-molecule inhibitors to target hepatic macrophages for the treatment of liver diseases [43]. The major advantages of these delivery vehicles are improved stability, site-specific delivery, and thus increased pharmacokinetics of the encapsulated therapeutics [44].

\section{miRNA/siRNA-Based Approaches}

The increasing understanding of the role of molecular pathways in pathogenesis of many diseases has given rise to in- depth research for strategies to manipulate the expression of specific genes that are involved in liver pathogenesis. Gene expression is biologically regulated via RNA interference, wherein microRNAs (miRNAs) and small interfering RNAs (siRNAs) are fundamental. miRNAs and siRNAs are naturally occurring small non-coding RNA molecules composed of about 22 nucleotides that regulate gene expression via gene silencing at the posttranscriptional level [42, 43]. New insights illustrate that miRNA and siRNA expression is specifically altered in nearly all liver diseases, suggesting the great involvement of dysregulation of these non-coding RNAs in liver pathology $[45,46]$. Therefore, extensive research has been performed into the possibilities of miRNAs and siRNAs to serve as a therapeutic target, whereby both inhibition and overexpression of specific miRNAs and siRNAs are therapeutically in reach [47]. However, the therapeutic approaches of miRNA and siRNA are quite different. This is mainly because miRNA is known to have multiple targets, whereas siRNA is highly specific with only one mRNA target [48]. MomenHeravi et al. showed in vitro that EVs can successfully deliver a miR-155 inhibitor to macrophages. miR-155 is known to be involved in liver inflammation and fibrogenesis, and delivery of a miR-155 inhibitor to RAW macrophages has shown to cause $>50 \%$ suppression of LPS-induced tumor necrosis factor alpha (TNF- $\alpha$ ) production, a critical pro-inflammatory mediator involved in liver diseases [27, 48, 49]. He et al. have investigated the potential of mannose-modified trimethyl chitosan-cysteine (MTC)-conjugated NPs containing TNF- $\alpha$ siRNA for targeted inhibition of TNF- $\alpha$ expression by macrophages. Intriguingly, in vitro studies using RAW macrophages showed $\sim 70 \%$ TNF- $\alpha$ silencing efficiency, requiring only $0.15 \mathrm{pm} / \mathrm{well}$ siRNA. These results were confirmed in an in vivo study, whereby orally delivered MTC NPs that contained a small amount of TNF- $\alpha$ siRNA inhibited TNF- $\alpha$ production in macrophages and protected mice from liver inflammation and damage caused by acute hepatic injury [28].

Beside using miRNA/siRNA to inhibit the secretion of proinflammatory mediators such as TNF- $\alpha$ by macrophages, there is another strategy that involves the inhibition of recruitment of circulating monocytes-derived macrophages. Upon liver injury, hepatic recruitment of MoMFs is mainly driven by $\mathrm{C}-\mathrm{C}$ motif chemokine receptor/ligand interactions such as CCR2/CCL2 [7, 50, 51]. Since the infiltration of MoMFs contributes to inflammatory processes and thus to the progression of liver diseases, obstructing chemokine signaling can be an attractive potential therapeutic target to dampen the recruitment of monocytes into the liver [52]. Kim et al. showed that silencing the expression of CCR2 through delivery of antiCCR2 siRNA to RAW macrophages induced $\sim 60 \%$ reduction in CCR2 expression levels. Similar effects were observed in vivo in diet-induced obese mice, which showed decreased numbers of infiltrated monocytes into the injured liver. In 
Table 1 Therapeutic targeting strategies of hepatic macrophages*

\begin{tabular}{|c|c|c|c|}
\hline Strategy & (nano) Therapeutics & Mechanism & Reference(s) \\
\hline \multirow{3}{*}{$\begin{array}{l}\text { miRNA/siRNA-based } \\
\text { approaches }\end{array}$} & EV-based delivery of miR-155 inhibitor & Inhibition of $\mathrm{KC}$ activation & (Momen-Heravi et al., 2014) [27] \\
\hline & $\begin{array}{l}\text { Mannose-modified trimethyl chitosan-cysteine } \\
\text { (MTC)-TNF } \alpha \text { siRNA NPs }\end{array}$ & Inhibition of $\mathrm{KC}$ activation & (He et al., 2013) [28] \\
\hline & Anti-CCR2 siRNA & Dampening of monocyte recruitment & (Kim et al., 2016) [29] \\
\hline \multirow[t]{3}{*}{$\begin{array}{r}\text { Protein-based } \\
\text { approaches }\end{array}$} & $\begin{array}{l}\text { Partially humanized anti-HMGB1 monoclonal } \\
\text { antibody }\end{array}$ & $\begin{array}{l}\text { Inhibition of } \mathrm{KC} \text { activation, dampening of } \\
\text { monocyte recruitment }\end{array}$ & (Lundbäck et al., 2016) [30] \\
\hline & $\begin{array}{l}\text { IL-4/anti-IL-4 monoclonal antibody complex } \\
\text { (IL-4c) }\end{array}$ & Modulation of macrophage polarization & (Lynch et al., 2019) [31] \\
\hline & Human monoclonal antibody BTT-1023 & Dampening of monocyte recruitment & (Arndtz et al., 2017) [32] \\
\hline \multirow{7}{*}{$\begin{array}{l}\text { Small-molecule } \\
\text { inhibitors }\end{array}$} & ASK1 inhibitor selonsertib & Inhibition of $\mathrm{KC}$ activation & (Loomba et al., 2018) [33] \\
\hline & NIK inhibitor B022 & Inhibition of $\mathrm{KC}$ activation & (Xiaomeng et al., 2016) [34] \\
\hline & CCR2/CCR5 antagonist cenicriviroc & Dampening of monocyte recruitment & $\begin{array}{l}\text { (Lefebvre et al., 2016; Krenkel } \\
\quad \text { et al., 2018) }[35,36]\end{array}$ \\
\hline & CTD-002 & $\begin{array}{l}\text { Inhibition of KC activation, dampening of } \\
\text { monocyte recruitment }\end{array}$ & (Khurana et al., 2019) [37] \\
\hline & SYK pathway inhibitor R406 & $\begin{array}{l}\text { Inhibition of KC activation, dampening of } \\
\text { monocyte recruitment }\end{array}$ & (Bukong et al., 2016) [38] \\
\hline & R406-PLGA NPs & $\begin{array}{l}\text { Inhibition of } \mathrm{KC} \text { activation, dampening of } \\
\text { monocyte recruitment }\end{array}$ & (Kurniawan et al., 2018) [39] \\
\hline & Docosahexanoic acid (DHA) & Modulation of macrophage polarization & (Carpino et al., 2016) [40] \\
\hline
\end{tabular}

*Abbreviations used are ASK-1, apoptosis signal-regulating kinase 1; CCR2, chemokine (C-C motif) receptor 2; CCR5, chemokine (C-C motif) receptor 5; CTD, cathepsin D; DHA, docosahexanoic acid; EV, extracellular vesicle; HMGB1, high mobility group box 1; KC, Kupffer cell(s); MTC, mannosemodified trimethly chitosan-cysteine; NF-kB, nuclear factor kappa-light-chain-enhancer of activated B cells; NIK, NF-kB-inducing kinase; NPs, nanoparticles; PLGA, poly(lactic-co-glycolic acid); SYK, spleen tyrosine kinase

addition, $\sim 70 \%$ reduction in TNF- $\alpha$ levels and complete reversal of steatosis was evidenced [29]. These results indicate that strategies comprising cell-targeted gene knockdown through miRNA/siRNA are promising therapeutic approaches for the treatment of various liver diseases. However, the implementation of these two RNA molecules as novel therapeutics currently face some limitations with regard to clinical translation due to potential off-target effects, delivery efficacy, and poor stability in vivo. However with successful implementation of Onpattro, first siRNA-based lipid nanoparticles for the treatment of hereditary transthyretin amyloidosis [53], siRNA/miRNA-based (nano) therapeutics offer exciting and daring platforms for scientists to overcome these challenges.

\section{Protein-Based Approaches}

Proteins, especially monoclonal antibodies, and peptides are highly promising therapeutics due to their high biocompatibility, non-toxicity, biodegradability, and selectivity [54]. Nowadays, monoclonal antibodies are receiving more and more attention from scientists to serve as therapeutic agents due to their specificity and capability of blocking the activity of specific signaling molecules, cell surface markers, or enzymes [55, 56]. Lundbäck and co-workers explored the therapeutic application of a partially humanized anti-high mobility group box 1 (HMGB1) monoclonal antibody in drug-induced liver injury (DILI) and ALF.
HMGB1 is one of the most well-known damage-associated molecular patterns (DAMPS), and the release of HMGB1 by macrophages and other immune cells is correlated with liver injury and inflammation with poor prognosis. Interestingly, mice treated with HMGB1-neutralizing antibody showed reduced liver injury and inflammation [30, 57]. More recently, Lynch et al. reported that administration of IL-4/anti-IL-4 monoclonal antibody complexes (IL-4c) reduced the number of pro-inflammatory macrophages in C57Bl/6 mice with acute liver injury. IL-4c therefore were capable of fostering liver regeneration upon acute injury through promoting a switch from macrophages with a pathogenic phenotype towards macrophages with a restorative phenotype [31].

Furthermore, human monoclonal antibody BTT-1023, also known as Timolumab that targets vascular adhesion protein-1 (VAP-1), is currently examined as novel treatment option for the progressive inflammatory liver disease, primary sclerosing cholangitis (PSC). VAP-1 is an endothelial glycoprotein that is highly implicated in leukocyte migration towards the inflamed site, thereby driving inflammation and fibrosis. Blockade of VAP-1 via BTT-1023 can therefore be an interesting approach to attenuate leukocyte trafficking and thus inhibit inflammatory responses. At present, BTT-1023 is intensively studied to determine its safety, dose, and efficacy $[32,58,59]$. 


\section{Small-Molecule Inhibitors}

Small-molecule inhibitors are compounds with low molecular weight $(<1000 \mathrm{Da})$, which allows them to translocate through cell membranes to reach and interact with intracellular targets to disrupt specific pathological pathways [60]. Since hepatic macrophages are known to be regulated by multiple inflammatory signaling pathways including apoptosis signal-regulating kinase 1 (ASK1) and nuclear factor kappa-light-chain-enhancer of activated $\mathrm{B}$ cells (NF-KB) [61], small-molecule inhibitors targeting these signaling pathways have been developed and explored for the treatment of liver diseases. Loomba and co-workers has evaluated a small-molecule ASK1 inhibitor, selonsertib, which has shown to inhibit hepatocyte metabolism and macrophage activation. In patients with NASH and fibrosis (randomized phase 2 trial), selonsertib evidenced an improvement in fibrosis, lobular inflammation, and serum biomarkers of apoptosis and necrosis [33], while in STELLAR-3 and STELLAR-4 randomized double-blind placebo-controlled phase 3 trials in NASH patients with F3 fibrosis and F4 cirrhosis, respectively, selonsertib failed to show a significant improvement in fibrosis. In another study, Xiaomeng et al. found a potent small-molecule inhibitor (B022) that efficiently inhibits NF-KB-inducing kinase (NIK). Inhibiting this inflammatory NIK pathway through B022 has shown to ameliorate macrophage-induced liver inflammation, as macrophages (mainly KCs) are strong activators of NF-kB [34, 62].

Another strategy besides inhibition of macrophage activation via specific inflammatory signaling pathways involves, as mentioned earlier, the dampening of inflammatory monocyte recruitment via CCR2/CCR5 [15]. Studies have demonstrated that blocking of CCL2-mediated monocyte recruitment using a selective CCR2/CCR5 small-molecule inhibitor, cenicriviroc, ameliorates inflammation, and fibrosis in the liver and kidney fibrosis mouse models [35, 36]. Interestingly, Friedman et al., in patients with NASH (NAFLD activity score, NAS $\geq 4$ ) and stage 1-3 liver fibrosis, has shown $\geq 2$-point improvement in NAS and $\geq 1$ stage improvement in fibrosis with favorable safety and tolerability after 1-year treatment with cenicriviroc in a CENTAUR phase 2b clinical study [63].

Khurana and co-workers investigated a highly specific small-molecule inhibitor CTD-002 of extracellular cathepsin $\mathrm{D}$ (CTSD), a lysosomal enzyme that is known to be associated with lipid-related disorders such as NAFLD. In this study, bone marrow-derived macrophages (BMDMs) were treated with CTD-002 and incubation with CTD-002-treated BMDMs-conditioned medium on human hepatocellular carcinoma (HepG2) cells resulted in reduced levels of inflammation and improved cholesterol metabolism. In addition, high fat diet-fed rats treated with CTD-002 showed attenuated hepatic steatosis [37]. Bukong et al., and Kurniawan and colleagues demonstrated the potential of a small-molecule inhibitor (R406) that inhibits spleen tyrosine kinase (SYK) [38, 39]. SYK signaling pathway has been shown to play an important role in the pathogenesis of alcoholic and non-alcoholic steatohepatitis driving liver inflammation and steatosis [38, 39]. SYK inhibitor, R406 ameliorated hepatic injury, steatosis and inflammation in alcoholic steatohepatitis in mice by inhibition of recruitment of immune cells macrophage and inflammasome activation [38]. R406, when delivered via poly(lactic-co-glycolic acid) (PLGA) NPs, showed significant inhibition of M1-specific inflammatory markers in RAW and BMDMs in vitro and attenuated liver inflammation, fibrosis, and steatosis in vivo in methionine-choline-deficient (MCD) diet-induced NASH mouse model [39].

Furthermore, Carpino et al. demonstrated that docosahexanoic acid (DHA), an omega-3 fatty acid, abrogated pediatric NAFLD through the interaction with G proteincoupled receptor 120 (GPR120) and reduced expression of NF-KB. GPR120 is known to be involved in macrophage polarization towards the M1/M2 phenotype. Treatment with DHA resulted in decreased polarization of macrophages towards the inflammatory M1 phenotype and increased polarization towards M2 macrophages, along with the improvement in NAS and reduction in hepatic progenitor cell activation, thereby suppressing inflammatory responses and fostering tissue repair respectively [40, 64].

\section{Conclusions}

Our improved fundamental understanding about the initiation, progression, and resolution of liver diseases has provided us the tools for the development of promising therapeutic approaches for the treatment of liver diseases. Hepatic macrophages play an essential role in maintaining liver homeostasis; however, numerous studies have found that when imbalance in their functioning occurs owing to liver injury, they are also involved in the initiation and progression of different etiological liver diseases. Intriguingly, hepatic macrophages possess tremendous heterogeneity and therefore possess diverse phenotypes with discrete functions in the liver. In the past decades, multiple approaches have been developed to target hepatic macrophages for the treatment of liver diseases. These approaches mainly aim at inhibiting $\mathrm{KC}$ activation, influencing macrophage polarization, and inhibiting monocyte infiltration, and great progress is being made in the application of specifically modified EVs and synthetic NPs for the delivery of therapeutics. As described in this review, innovative therapeutic strategies entail the application of miRNA/siRNA to alter expression levels of specific genes that are correlated with disease progression. Another strategy involves proteinbased macrophage targeting, wherein monoclonal antibodies 
appear as increasingly interesting therapeutic agents due to their high specificity. Small-molecule inhibitors are the most extensively studied therapeutics, and plenty of them are currently advancing to clinical trials. Nevertheless, also miRNA/ siRNA- and protein-based approaches have already shown promising results from in vitro studies and in experimental animal models. However, there are still several obstacles that are hampering the translation from animal models to the clinic, e.g., disparity in macrophage phenotypes and the fact that there is larger macrophage heterogeneity in humans in comparison with animal models. Furthermore, it still remains not fully understood, yet a subject of intensive research, what the underlying mechanisms of hepatic macrophage phenotype switching are during the diverse stages of liver diseases. Therefore, in-depth investigation of distinct macrophage phenotypes and their exact role in liver diseases is required to help unravel the complex liver pathobiology and to develop novel therapeutic targets to treat liver diseases in the clinic.

\section{Compliance with Ethical Standards}

Conflict of Interest Nijland and Dr. Bansal declare that they have no conflicts of interest.

Human and Animal Rights and Informed Consent This article does not contain any studies with human or animal subjects performed by any of the authors.

Open Access This article is licensed under a Creative Commons Attribution 4.0 International License, which permits use, sharing, adaptation, distribution and reproduction in any medium or format, as long as you give appropriate credit to the original author(s) and the source, provide a link to the Creative Commons licence, and indicate if changes were made. The images or other third party material in this article are included in the article's Creative Commons licence, unless indicated otherwise in a credit line to the material. If material is not included in the article's Creative Commons licence and your intended use is not permitted by statutory regulation or exceeds the permitted use, you will need to obtain permission directly from the copyright holder. To view a copy of this licence, visit http://creativecommons.org/licenses/by/4.0/.

\section{References}

1. Robinson MW, Harmon C, O'Farrelly C. Liver immunology and its role in inflammation and homeostasis. Cell Mol Immunol. 2016;13(3):267-76. https://doi.org/10.1038/cmi.2016.3.

2. van der Heide D, Weiskirchen R, Bansal R. Therapeutic targeting of hepatic macrophages for the treatment of liver diseases. Front Immunol. 2019;10(2852). doi:https://doi.org/10.3389/fimmu. 2019.02852 .

3. Jenne CN, Kubes P. Immune surveillance by the liver. Nat Immunol. 2013;14(10):996-1006. https://doi.org/10.1038/ni.2691.

4. Tacke F, Zimmermann HW. Macrophage heterogeneity in liver injury and fibrosis. J Hepatol. 2014;60(5):1090-6. https://doi.org/10. 1016/j.jhep.2013.12.025.
5. Gordon S, Pluddemann A, Martinez EF. Macrophage heterogeneity in tissues: phenotypic diversity and functions. Immunol Rev. 2014;262(1):36-55. https://doi.org/10.1111/imr.12223.

6. Guillot A, Tacke F. Liver macrophages: old dogmas and new insights. Hepatol Commun. 2019;3(6):730-43. https://doi.org/10. 1002/hep4.1356.

7. Ju C, Tacke F. Hepatic macrophages in homeostasis and liver diseases: from pathogenesis to novel therapeutic strategies. Cell Mol Immunol. 2016;13(3):316-27. https://doi.org/10.1038/cmi.2015. 104.

8. Krenkel O, Tacke F. Liver macrophages in tissue homeostasis and disease. Nat Rev Immunol. 2017;17:306-21. https://doi.org/10. 1038/nri.2017.11.

9. Seo W, Gao Y, He Y, Sun J, Xu H, Feng D, et al. ALDH2 deficiency promotes alcohol-associated liver cancer by activating oncogenic pathways via oxidized DNA-enriched extracellular vesicles. J Hepatol. 2019;71(5):1000-11. https://doi.org/10.1016/j.jhep.2019. 06.018 .

10. Geerts A. History, heterogeneity, developmental biology, and functions of quiescent hepatic stellate cells. Semin Liver Dis. 2001;21: 311-35. https://doi.org/10.1055/s-2001-17550.

11. Rombouts K. Chapter 2 - hepatic stellate cell culture models. In: Gandhi CR, Pinzani M, editors. Stellate cells in health and disease. Boston: Academic Press; 2015. p. 15-27.

12. Hellerbrand C. Hepatic stellate cells-the pericytes in the liver. Pflugers Arch. 2013;465(6):775-8. https://doi.org/10.1007/ s00424-012-1209-5.

13. Pradere JP, Kluwe J, De Minicis S, Jiao JJ, Gwak GY, Dapito DH, et al. Hepatic macrophages but not dendritic cells contribute to liver fibrosis by promoting the survival of activated hepatic stellate cells in mice. Hepatology (Baltimore, Md). 2013;58(4):1461-73. https:// doi.org/10.1002/hep.26429.

14. Dong X, Liu J, Xu Y, Cao H. Role of macrophages in experimental liver injury and repair in mice. Exp Ther Med. 2019;17(5):383547. https://doi.org/10.3892/etm.2019.7450.

15. Tacke F. Targeting hepatic macrophages to treat liver diseases. J Hepatol. 2017;66(6):1300-12. https://doi.org/10.1016/j.jhep.2017. 02.026 .

16. Wang J, Kubes P. A reservoir of mature cavity macrophages that can rapidly invade visceral organs to affect tissue repair. Cell. 2016;165(3):668-78. https://doi.org/10.1016/j.cell.2016.03.009.

17. Bilzer M, Roggel F, Gerbes A. Role of Kupffer cells in host defence and liver disease. Liver Int. 2006;26(10):1175-86.

18. Orecchioni M, Ghosheh Y, Pramod AB, Ley K. Macrophage polarization: different gene signatures in M1(LPS+) vs. classically and M2(LPS-) vs. alternatively activated macrophages. Front Immunol. 2019;10:1084.

19. Sica A, Invernizzi P, Mantovani A. Macrophage plasticity and polarization in liver homeostasis and pathology. Hepatology (Baltimore, Md). 2014;59(5):2034-42. https://doi.org/10.1002/ hep.26754.

20. Martinez FO, Sica A, Mantovani A, Locati M. Macrophage activation and polarization. Front Biosci. 2008;13:453-61.

21. Roszer T. Understanding the mysterious M2 macrophage through activation markers and effector mechanisms. Mediat Inflamm. 2015;2015:816460. https://doi.org/10.1155/2015/816460.

22. Mantovani A, Sica A, Sozzani S, Allavena P, Vecchi A, Locati M. The chemokine system in diverse forms of macrophage activation and polarization. Trends Immunol. 2004;25(12):677-86. https:// doi.org/10.1016/j.it.2004.09.015.

23. Asrani SK, Devarbhavi H, Eaton J, Kamath PS. Burden of liver diseases in the world. J Hepatol. 2019;70(1):151-71. https://doi. org/10.1016/j.jhep.2018.09.014.

24. Weiskirchen R, Weiskirchen S, Tacke F. Recent advances in understanding liver fibrosis: bridging basic science and individualized 
treatment concepts. F1000Res. 2018;7:F1000 Faculty Rev-921. https://doi.org/10.12688/f1000research.14841.1.

25. Friedman SL. Mechanisms of hepatic Fibrogenesis. Gastroenterology. 2008;134(6):1655-69. https://doi.org/10.1053/ j.gastro.2008.03.003.

26. Chang J, Hisamatsu T, Shimamura K, Yoneno K, Adachi M, Naruse $\mathrm{H}$, et al. Activated hepatic stellate cells mediate the differentiation of macrophages. Hepatol Res. 2013;43(6):658-69. https:// doi.org/10.1111/j.1872-034X.2012.01111.x.

27. Momen-Heravi F, Bala S, Bukong T, Szabo G. Exosome-mediated delivery of functionally active miRNA-155 inhibitor to macrophages. Nanomedicine. 2014;10(7):1517-27. https://doi.org/10. 1016/j.nano.2014.03.014.

28. He C, Yin L, Tang C, Yin C. Multifunctional polymeric nanoparticles for oral delivery of TNF- $\alpha$ siRNA to macrophages. Biomaterials. 2013;34(11):2843-54. https://doi.org/10.1016/j. biomaterials.2013.01.033.

29. Kim J, Chung K, Choi C, Beloor J, Ullah I, Kim N, et al. Silencing CCR2 in macrophages alleviates adipose tissue inflammation and the associated metabolic syndrome in dietary obese mice. Mol Ther Nucleic Acids. 2016;5(1):e280-e. https://doi.org/10.1038/mtna. 2015.51.

30. Lundbäck P, Lea JD, Sowinska A, Ottosson L, Fürst CM, Steen J, et al. A novel high mobility group box 1 neutralizing chimeric antibody attenuates drug-induced liver injury and postinjury inflammation in mice. Hepatology (Baltimore, Md). 2016;64(5):1699710. https://doi.org/10.1002/hep.28736.

31. Lynch R, Bain C, Hawley C, Forbes S, Jenkins S. SAT-375therapeutic interleukin 4 modulates monocyte dynamics and accelerates repair and regeneration following acute liver injury. J Hepatol. 2019;70(1):e800. https://doi.org/10.1016/S06188278(19)31595-6.

32. Arndtz K, Corrigan M, Rowe A, Kirkham A, Barton D, Fox R, et al. Investigating the safety and activity of the use of BTT1023 (Timolumab), in the treatment of patients with primary sclerosing cholangitis (BUTEO): a single-arm, two-stage, open-label, multicentre, phase II clinical trial protocol. BMJ Open. 2017;7:e015081. https://doi.org/10.1136/bmjopen-2016-015081.

33. Loomba R, Lawitz E, Mantry PS, Jayakumar S, Caldwell SH, Arnold $\mathrm{H}$, et al. The ASK1 inhibitor selonsertib in patients with nonalcoholic steatohepatitis: a randomized, phase 2 trial. Hepatology. 2018;67(2):549-59. https://doi.org/10.1002/hep. 29514.

34. Xiaomeng R, Xinzhi L, Jia L, Chen D, Hou H, Rui L et al. A smallmolecule inhibitor of NF- B-inducing kinase (NIK) protects liver from toxin-induced inflammation, oxidative stress, and injury. FASEB J 2016;31. doi:https://doi.org/10.1096/fj.201600840R.

35. Lefebvre E, Moyle G, Reshef R, Richman LP, Thompson M, Hong $\mathrm{F}$, et al. Antifibrotic effects of the dual CCR2/CCR5 antagonist cenicriviroc in animal models of liver and kidney fibrosis. PLoS One. 2016;11(6):e0158156-e. https://doi.org/10.1371/journal. pone. 0158156 .

36. Krenkel O, Puengel T, Govaere O, Abdallah AT, Mossanen JC, Kohlhepp M, et al. Therapeutic inhibition of inflammatory monocyte recruitment reduces steatohepatitis and liver fibrosis. Hepatology. 2018;67(4):1270-83. https://doi.org/10.1002/hep. 29544.

37. Khurana P, Yadati T, Goyal S, Dolas A, Houben T, Oligschlaeger Y, et al. Inhibiting extracellular cathepsin D reduces hepatic steatosis in Sprague Dawley rats (†). Biomolecules. 2019;9(5):171. https:// doi.org/10.3390/biom9050171.

38. Bukong TN, Iracheta-Vellve A, Saha B, Ambade A, Satishchandran A, Gyongyosi B, et al. Inhibition of spleen tyrosine kinase activation ameliorates inflammation, cell death, and steatosis in alcoholic liver disease. Hepatology. 2016;64(4):1057-71. https://doi.org/10. 1002/hep.28680.
39. Kurniawan DW, Jajoriya AK, Dhawan G, Mishra D, Argemi J, Bataller R, et al. Therapeutic inhibition of spleen tyrosine kinase in inflammatory macrophages using PLGA nanoparticles for the treatment of non-alcoholic steatohepatitis. J Control Release. 2018;288:227-38. https://doi.org/10.1016/j.jconrel.2018.09.004.

40. Carpino G, Nobili V, Renzi A, Stefanis C, Stronati L, Franchitto A, et al. Macrophage activation in pediatric nonalcoholic fatty liver disease (NAFLD) correlates with hepatic progenitor cell response via Wnt3a pathway. PLoS One. 2016;11:e0157246. https://doi.org/ 10.1371/journal.pone.0157246.

41. Rizvi SAA, Saleh AM. Applications of nanoparticle systems in drug delivery technology. Saudi Pharm J. 2018;26(1):64-70. https://doi.org/10.1016/j.jsps.2017.10.012.

42. Raposo G, Stahl PD. Extracellular vesicles: a new communication paradigm? Nat Rev Mol Cell Biol. 2019;20:509-10.

43. Deng F, Magee N, Zhang Y. Decoding the role of extracellular vesicles in liver diseases. Liver Res. 2017;1(3):147-55. https:// doi.org/10.1016/j.livres.2017.11.003.

44. Din FU, Aman W, Ullah I, Qureshi OS, Mustapha O, Shafique S, et al. Effective use of nanocarriers as drug delivery systems for the treatment of selected tumors. Int J Nanomedicine. 2017;12:7291309. https://doi.org/10.2147/IJN.S146315.

45. Schueller F, Roy S, Vucur M, Trautwein C, Luedde T, Roderburg C. The role of miRNAs in the pathophysiology of liver diseases and toxicity. Int J Mol Sci. 2018;19(1):261. https://doi.org/10.3390/ ijms 19010261.

46. Lai C, Higueras C, Salido E. siRNA therapeutics to treat liver disorders. 2017. p. 159-90.

47. Szabo G, Bala S. MicroRNAs in liver disease. Nat Rev Gastroenterol Hepatol. 2013;10(9):542-52. https://doi.org/10. 1038/nrgastro.2013.87.

48. Lam JKW, Chow MYT, Zhang Y, Leung SWS. siRNA versus miRNA as therapeutics for gene silencing. Mol Ther Nucleic Acids. 2015;4:e252. https://doi.org/10.1038/mtna.2015.23.

49. Zhang W, Zhou Y, Li X, Xu X, Chen Y, Zhu R, et al. Macrophagetargeting and reactive oxygen species (ROS)-responsive nanopolyplexes mediate anti-inflammatory siRNA delivery against acute liver failure (ALF). Biomater Sci. 2018;6(7):1986-93. https:// doi.org/10.1039/C8BM00389K.

50. Cha J-Y, Kim D-H, Chun K-H. The role of hepatic macrophages in nonalcoholic fatty liver disease and nonalcoholic steatohepatitis. Lab Anim Res. 2018;34(4):133-9. https://doi.org/10.5625/lar. 2018.34.4.133.

51. Lefere S, Tacke F. Macrophages in obesity and non-alcoholic fatty liver disease: crosstalk with metabolism. JHEP Rep. 2019;1(1):30 43. https://doi.org/10.1016/j.jhepr.2019.02.004.

52. Chen W, Zhang J, Fan H, Zhu J. Function and therapeutic advances of chemokine and its receptor in nonalcoholic fatty liver disease. Ther Adv Gastroenterol 2018;11.

53. Akinc A, Maier MA, Manoharan M, Fitzgerald K, Jayaraman M, Barros S, et al. The Onpattro story and the clinical translation of nanomedicines containing nucleic acid-based drugs. Nat Nanotechnol. 2019;14(12):1084-7. https://doi.org/10.1038/ s41565-019-0591-y.

54. Araste F, Abnous K, Hashemi M, Taghdisi SM, Ramezani M, Alibolandi M. Peptide-based targeted therapeutics: focus on cancer treatment. J Control Release. 2018;292:141-62. https://doi.org/10. 1016/j.jconrel.2018.11.004.

55. Shepard HM, Phillips GL, D Thanos C, Feldmann M. Developments in therapy with monoclonal antibodies and related proteins. Clin Med (Lond). 2017;17(3):220-32. https://doi.org/10. 7861/clinmedicine.17-3-220.

56. Awwad S, Angkawinitwong U. Overview of antibody drug delivery. Pharmaceutics. 2018;10(3):83. https://doi.org/10.3390/ pharmaceutics 10030083 . 
57. Triantafyllou E, Woollard KJ, McPhail MJW, Antoniades CG, Possamai LA. The role of monocytes and macrophages in acute and acute-on-chronic liver failure. Front Immunol. 2018;9:2948. https://doi.org/10.3389/fimmu.2018.02948.

58. Weston CJ, Shepherd EL, Claridge LC, Rantakari P, Curbishley SM, Tomlinson JW, et al. Vascular adhesion protein-1 promotes liver inflammation and drives hepatic fibrosis. J Clin Invest. 2015;125(2):501-20. https://doi.org/10.1172/JCI73722.

59. Autio A, Vainio P, Suilamo S, Mali A, Vainio J, Saanijoki T et al. Preclinical evaluation of a radioiodinated fully human antibody for in vivo imaging of vascular adhesion protein-1-positive vasculature in inflammation. J Nucl Med 2013;54. doi:https://doi.org/10.2967/ jnumed.113.120295.

60. Bansal R, Nagórniewicz B, Prakash J. Clinical Advancements in the Targeted Therapies against Liver Fibrosis. Mediat Inflamm. 2016;2016:16. https://doi.org/10.1155/2016/7629724.

61. Weiskirchen R, Tacke F. Liver fibrosis: from pathogenesis to novel therapies. Dig Dis. 2016;34(4):410-22. https://doi.org/10.1159/ 000444556.
62. Czauderna C, Castven D, Mahn FL, Marquardt JU. Contextdependent role of NF-kappaB signaling in primary liver cancerfrom tumor development to therapeutic implications. Cancers. 2019;11(8). doi:https://doi.org/10.3390/cancers11081053.

63. Friedman SL, Ratziu V, Harrison SA, Abdelmalek MF, Aithal GP, Caballeria J, et al. A randomized, placebo-controlled trial of cenicriviroc for treatment of nonalcoholic steatohepatitis with fibrosis. Hepatology. 2018;67(5):1754-67. https://doi.org/10.1002/hep. 29477.

64. Alisi A, Carpino G, de Oliveira F, Panera N, Nobili V, Gaudio E. The role of tissue macrophage-mediated inflammation on NAFLD pathogenesis and its clinical implications. Mediat Inflamm. 2017;2017:1-15. https://doi.org/10.1155/2017/8162421.

Publisher's Note Springer Nature remains neutral with regard to jurisdictional claims in published maps and institutional affiliations. 